\title{
Emergency Vaginal myomectomy of cervical leiomyoma in a pregnant woman at term.
}

\section{Dr. Shalini Malhotra ${ }^{1, ~ *}$ MD, MRCOG, Prof Dr. Osama Omran MD FRCOG}

${ }^{1}$ Consultants Obs. \& Gyn., Al Qassimi Women \& Children Hospital, Sharjah, UAE

*Corresponding Author: Dr. Shalini Malhotra, Consultants Obs. \& Gyn., Al Qassimi Women \& Children Hospital, Sharjah, UAE

Received date: 11 February 2021; Accepted date: 17 March 2021; Published date: 19 March 2021

Citation: Malhotra S, Emergency Vaginal myomectomy of cervical leiomyoma in a pregnant woman at term. J Med Case Rep Case Series 2(2): https://doi.org/10.38207/jmcrcs20210016

Copyright: ( 2021 Dr. Shalini Malhotra. This is an open-access article distributed under the terms of the Creative Commons Attribution License, which permits unrestricted use, distribution, and reproduction in any medium, provided the original author and source are credited.

Abstract
A case report of pedunculated cervical leiomyoma protruding from the vulva in a pregnant woman at 38 weeks of her gestational age. The
nature and the origin of this firm irreducible mass were difficult to assess in a term pregnant patient, as the patient was stressed in pain. It
needed examination under anesthesia to diagnose and plan appropriate management. The patient was worried about her pregnancy and
decided to be delivered at the same time. The mode of delivery was left for obstetrician by artificial rupture of membranes aiming for vaginal
delivery to proceed with a semi-elective cesarean section at the same set of examination under anesthesia.

\section{Introduction}

The prevalence of uterine leiomyomas in pregnancy is approximately $3 \%$ to $10 \%$. The prevalence of clinically evident cervical leiomyomas in pregnancy is less than $1 \%$ The prevalence of leiomyomas with pregnancy are reported as $3 \%$ to $10 \%$ approximately [1] While most pregnant women with fibroids do not have any complications during pregnancy related to the fibroids, about 1 in 10 have been presented with pain being the most common symptom. A slightly increased risk of obstetrical complications such as miscarriage, premature labor and delivery, abnormal fetal position, and placental abruption has also been reported. Quite a few times, the leiomyomas are discovered either due to pain or during routine

\section{Case Report}

Ms. K, Primigravida reported in an emergency at 37 weeks gestation age with a sudden history of a mass protruding out of vaginal introitus for the last few hours and not receding back. She gave a history of a similar mass protruding from the vagina off and on over the last two months, which was not painful, and spontaneously reduced after lying down and thus she ignored it and did not report it during her antenatal checkups with Consultant Obstetricians. The patient had her last on 5/820 at 9 pm, she attended Obstetrics \& Gynecology emergency department, as a case of 38 weeks pregnancy with a painful mass protruding from the vagina. No obstetric concerns were noted. The mass was approximate $9 \times 4 \mathrm{~cm}$ in size. It was distending the vaginal obstetric ultrasounds in early pregnancy.

Leiomyomas are benign monoclonal tumors arising from uterine smooth muscle cells and fibroblasts. They contain a large amount of extracellular matrix (including collagen, proteoglycan, fibronectin) and are surrounded by a thin pseudo capsule of areolar tissue and compressed muscle fibers. While the prevalence of clinically evident cervical leiomyoma in pregnancy is less than $1 \%$, it is rare to see one, acutely prolapsing outof the vagina in the third trimester of pregnancy. This case highlightsthe diagnostic dilemmas that we faced and the way we successfully managed the case.

period on $12^{\text {th }}$ November 2019 and thus her expected date of delivery was 19/8/20. She had consulted private doctors early in the index pregnancy and had only had one detailed anomaly scan at 21 weeks gestation. Neither OGTT nor early dating scan was done. She had visited our hospital twice in the second $\&$ third trimester of pregnancy and appropriate care was given. All booking investigations were within normal limits.

Introitus while protruding out of it. The origin of the mass could not be concluded upon as the patient was very un-cooperative due to extreme pain. It was pinkish in color, of firm consistency, and irreducible. (Picture 1) 


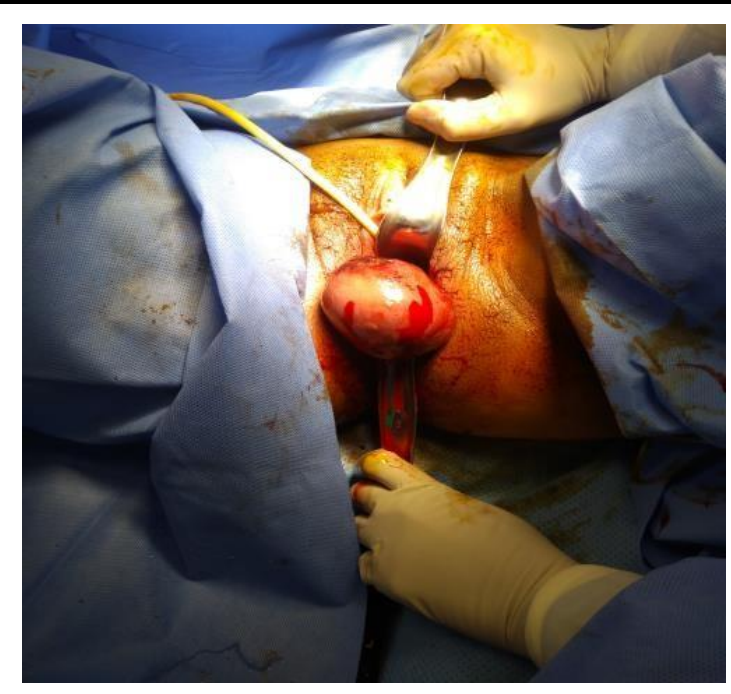

Picture 1: Mass distending introitus

The plan of management was discussed with the patient and her husband. They were explained that it was necessary to find out the nature of the mass and its origin, to decide about the way of management. Such an irreducible and painful mass, the assessment would be impossible to be completed except under anesthesia. Since she was 38 weeks pregnant, discussion regarding mode delivery was done including the dilemma regarding vaginal delivery by doing artificial rupture of membranes immediately after removal of the protruding mass, or to proceed for delivery by semi-elective cesarean section in the same sitting. Although the couple preferred to have a cesarean section, they left the final decision for a mode of delivery for the Obstetrician depending on the intra-operative findings, as they did not want to continue pregnancy any further. The consent has been taken with a detailed plan of management as agreed by the patient. Under regional anesthesia, vaginal examination revealed a large very vascular mass arising from the right side of the anterior lip of the cervix, separate from the vagina. It was found to be a cervical leiomyoma hanging down and protruding out of the vaginal introitus. The cervix was deformed and stenosis and impossible to do artificial rupture of membranes to induce her labor. once Cesarean delivery was done, a vaginal myomectomy was performed. The mass was approx. $9 \mathrm{~cm} \times 4 \mathrm{~cm}$ arising from the cervical anterior lip (Picture $2 \boldsymbol{\&}$ 3). This firm mass was enucleated from the well-defined capsule and redundant tissue was excised i.e., vaginal myomectomy. (Picture4) After ensuring hemostasis, the anterior lip of the cervix reconstructed with a no 2 Hegar dilator kept in the cervical canal to avoid its inadvertent closure during the repair.The patient was discharged well after 3 days of hospital stay and themother and baby are found to be doing well in the postnatal period. This cervical polyp was sent for histopathological examination and was found to be leiomyoma with degenerative changes with inflamed ectocervix.

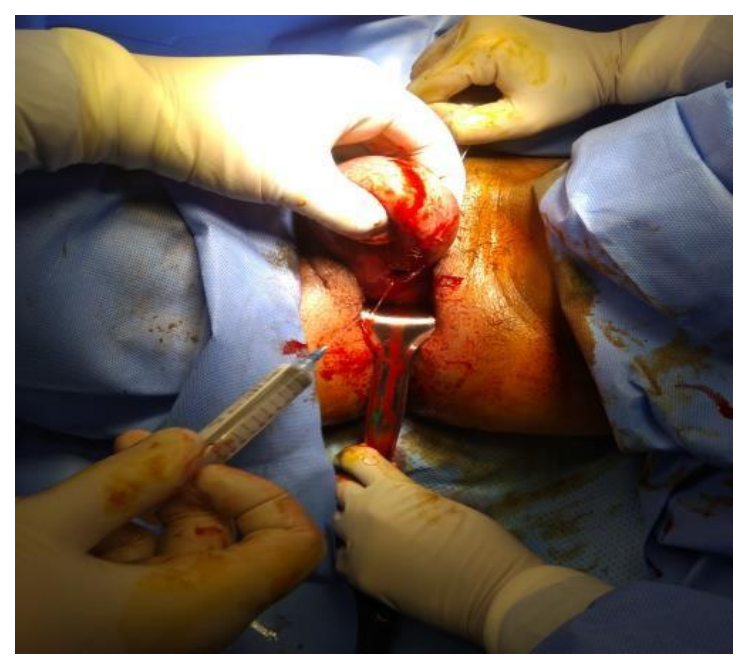

Picture 2: needle pointing to external os with mass anterior to it.

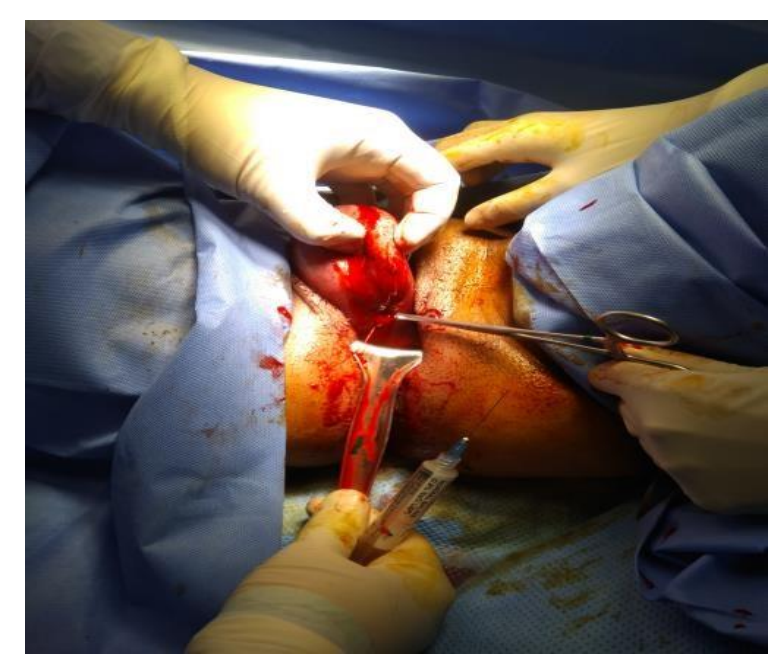

Picture 3: Allis forceps pointing to the external cervical os 


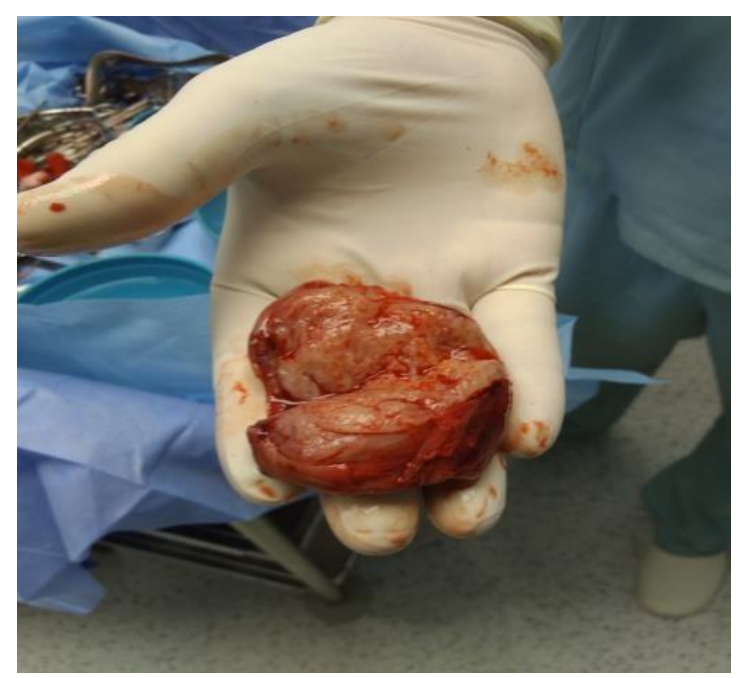

Picture 4: Cervical myoma-cut section sent for histopathology

\section{Discussion}

Even though we know, majority of uterine leiomyoma does not affect the life of most women, including uncomplicated situations during pregnancy, there are some occasions when patients need hospitalization. Cervical Leiomyomas can cause symptoms like bleeding, infection, excess vaginal discharges, pain, and urinary stasis thus may need surgical intervention like in the case described above and [3]. Most variants, being histologically defined, are diagnosed only after surgical removal, due to the inability of the clinician to imagine cervical fibroids as the cause.

As in this index case, the mass did not present as a typical leiomyoma. Even the location was so rare along with an atypical history (mass prolapsing and reducing during pregnancy with acute presentation of prolapse on admission). History of a mass, coming out on standing, reducing on lying down, most suggestive of a vaginal wall prolapse, though it is rare to see a vaginal wall prolapse becoming irreducible suddenly at 37 weeks pregnancy, leading to acute presentation and emergency admission. only after examination under anesthesia, it appeared to be a case of cervical leiomyoma, confirmed by a histopathological examination later. Cases of prolapsed leiomyoma in which pregnancy reached the term needed

\section{References}

1. Straub HL, Chohan L, Kilpatrick CC (2010) Cervical and prolapsed submucosal leiomyomas complicating pregnancy. Obstet Gynecol Surv. 65(9): 583-90.

2. Holdsworth-Carson SJ, Zaitseva M, Vollenhoven BJ, Rogers PA (2014) Clonality of smooth muscle and fibroblast cell populations isolated from human fibroid and myometrial tissues. Mol Hum Reprod 20(3): 250-9. emergency vaginal myomectomy are very rare. Previously, only three such cases have been reported, including the one written by Obara et al [4], where myomectomy was done at 13 weeks pregnancy and the lady still could reach term gestation. They did not report uterine contractions, short cervix, or cervical funneling in the remaining duration of pregnancy.

Vaginal myomectomy is recommended as the initial treatment of choice for a prolapsed, pedunculated submucous leiomyoma except when other indications necessitate an abdominal approach. The dilemma of doing a cesarean delivery meanwhile worth a debate. If the pregnancy continues till the spontaneous onset of labor, there is no evidence to suggest whether this cervix would go through labor processes (cervical dystocia) and enable the lady to have a vaginal delivery without the risk of intrapartum bleeding. Also, it is worth highlighting the possible risk of excessive bleeding during the vaginal myomectomy at 37 weeks gestation age which might compromise the fetal condition. Thus, counseling the patient and decisionmaking is challenging for the Obstetrician. The patient needs to understand the probabilities involved and the risks involved clearly before an informed decision is finalized and proceeded with for a good outcome.

3. Oruç S, Karaer O, Kurtul O (2004) Coexistence of a prolapsed, pedunculated cervical myoma and pregnancy complications: a case report. J Reprod Med. 49(7): 575-7.

4. Obara M, Hatakeyama Y, Shimizu Y (2014) Vaginal Myomectomy for Semipedunculated Cervical Myoma during Pregnancy. AJP Rep. 4(1): 37-40. 